\title{
Title
}

Respiratory Mechanics Assessment for Reverse-Triggered Breathing Cycles Using Pressure Reconstruction

\section{Authors list:}

Vincent Major ${ }^{\mathrm{a} *}$, Simon Corbett ${ }^{\mathrm{a}}$, Daniel Redmond ${ }^{\mathrm{a}}$, Alex Beatson ${ }^{\mathrm{a}}$, Daniel Glassenbury ${ }^{\mathrm{a}}$, Yeong Shiong Chiew ${ }^{a}$, Christopher Prettya ${ }^{a}$ Thomas Desaive ${ }^{b}$, Ákos Szlávecz ${ }^{c}$, Balázs Benyóc, Geoffrey M Shaw $^{\mathrm{d}}$ and J Geoffrey Chase ${ }^{\mathrm{a}}$

${ }^{a}$ Centre for Bioengineering, University of Canterbury, Christchurch, New Zealand

${ }^{\mathrm{b}}$ GIGA Cardiovascular Science, University of Liege, Liege, Belgium

${ }^{c}$ Department of Control Engineering and Information, Budapest University of Technology and Economics, Budapest, Hungary

${ }^{\mathrm{d}}$ Department of Intensive Care, Christchurch Hospital, Christchurch, New Zealand

\section{Contact details:}

Vincent Major - vincent.major65@gmail.com

Simon Corbett - simon.corbett14@gmail.com

Daniel Redmond - daniel.redmond@pg.canterbury.ac.nz

Alex Beatson - $\underline{\text { alexub@gmail.com }}$

Daniel Glassenbury - danielglassenbury@gmail.com

Yeong Shiong Chiew - yeongshiong.chiew@ canterbury.ac.nz

Chris Pretty - chris.pretty@ canterbury.ac.nz

Thomas Desaive - tdesaive@ulg.ac.be

Akos Szlavecz - szlavecz@iit.bme.hu

Balazs Benyo - bbenyo@iit.bme.hu

Geoffrey M Shaw - geoff.shaw@cdhb.health.nz

J Geoffrey Chase - geoff.chase@ canterbury.ac.nz

*Corresponding Authors

Vincent Major - vincent.major65@gmail.com 


\begin{abstract}
Monitoring patient-specific respiratory mechanics can be used to guide mechanical ventilation (MV) therapy in critically ill patients. However, many patients can exhibit spontaneous breathing (SB) efforts during ventilator supported breaths, altering airway pressure waveforms and hindering model-based (or other) identification of the true, underlying respiratory mechanics necessary to guide MV. This study aims to accurately assess respiratory mechanics for breathing cycles masked by SB efforts.
\end{abstract}

A cumulative pressure reconstruction method is used to ameliorate SB by identifying SB affected waveforms and reconstructing unaffected pressure waveforms for respiratory mechanics identification using a single-compartment model. Performance is compared to conventional identification without reconstruction, where identified values from reconstructed waveforms should be less variable. Results are validated with 9485 breaths affected by SB, including periods of muscle paralysis that eliminates $\mathrm{SB}$, as a validation test set where reconstruction should have no effect. In this analysis, the patients are their own control, with versus without reconstruction, as assessed by breath-to-breath variation using the non-parametric coefficient of variation $(\mathrm{CV})$ of respiratory mechanics.

Pressure reconstruction successfully estimates more consistent respiratory mechanics. CV of estimated respiratory elastance is reduced up to 78\% compared to conventional identification ( $\mathrm{p}<0.05$ ). Pressure reconstruction is comparable ( $\mathrm{p}>0.05$ ) to conventional identification during paralysis, and generally performs better as paralysis weakens, validating the algorithm's purpose.

Pressure reconstruction provides less-affected pressure waveforms, ameliorating the effect of SB, resulting in more accurate respiratory mechanics identification. Thus providing the opportunity to use respiratory mechanics to guide mechanical ventilation without additional muscle relaxants, simplifying clinical care and reducing risk.

Australian New Zealand Trial Registry Number: ACTRN12613001006730

Keywords: respiratory mechanics, decision support, mechanical ventilation, spontaneous breathing, reverse-triggering.

Abbreviations ${ }^{1}$ :

$\begin{array}{ll}\text { CV } & \text { Coefficient of Variation } \\ \text { MV } & \text { Mechanical Ventilation } \\ \text { PEEP } & \text { Positive End-Expiratory Pressure } \\ \text { SB } & \text { Spontaneous Breathing }\end{array}$ 


\subsection{Introduction}

Model-based methods to monitor respiratory mechanics for mechanical ventilation (MV) patients can assist clinicians to guide MV treatment [1-4]. However, true respiratory mechanics can be masked by spontaneous breathing (SB) efforts and cannot be estimated in these cases without the use of invasive measuring equipment or clinical protocols [5-7]. Since SB efforts can be common, the application of respiratory mechanics to guide MV remains limited [6].

Akoumianaki et al. [8] described a phenomenon where SB during volume controlled ventilation masks the true, measurable, respiratory system mechanics. This phenomenon is referred to as ventilatorinduced reverse-triggering of patient muscular breathing efforts. An example of the pressure waveform from a reverse-triggered breath is shown in Figure 1. The reverse-triggering or patient effort creates anomalies in the patient airway pressure waveform, resulting in potential mis-identification of underlying respiratory mechanics if using simple models [6,9]. Specifically, patient effort reduces the net airway pressure for a given volume and leads to a lower calculated respiratory elastance due to the effective negative elastance component resulting from the patient's inspiratory effort [10]. Hence, the identified parameters do not represent the true underlying mechanics, as the patient-specific, variable inspiratory effort input was not accounted for in the model.

In addition, the level of SB effort can be highly variable. While none may occur in any given breath, other subsequent breaths may be heavily or only lightly affected, as shown in Figure 1. Currently, modelling this input for real-time, breath-to-breath application is not possible, and direct measurements for later use, as with NAVA [11, 12] for example, are additionally invasive and costly and thus infeasible. Hence, there is a need to more easily mitigate these effects with a cost effective method without inducing further stress to patients. 
(a)

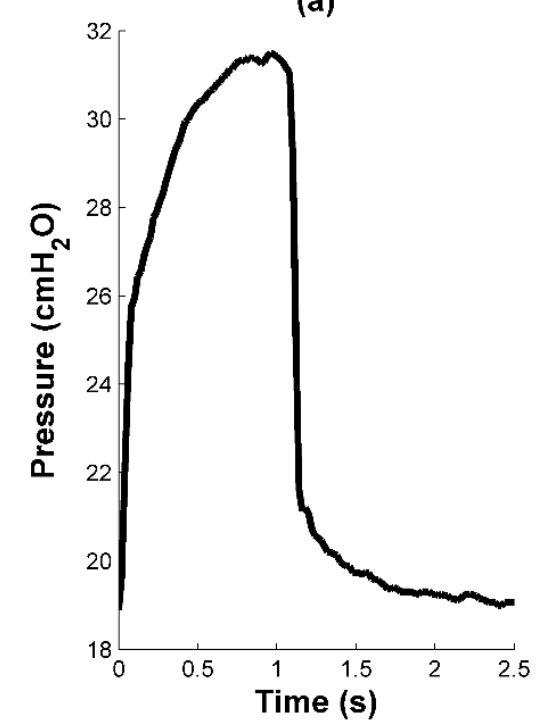

(b)

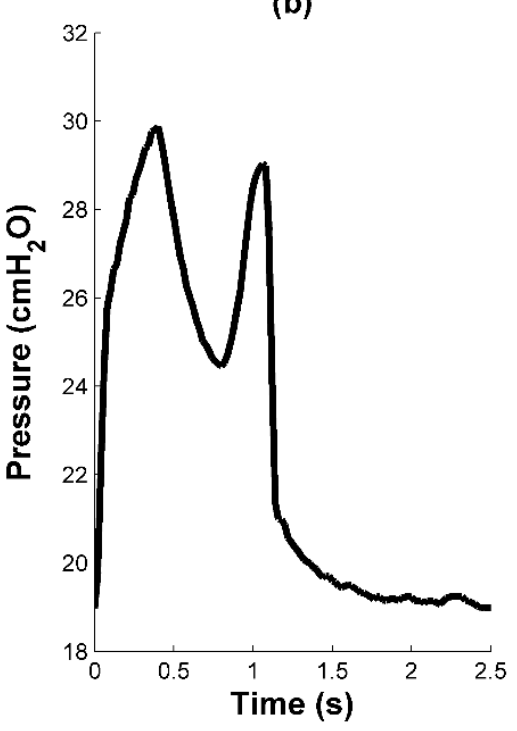

Figure 1. Comparison of a typical airway pressure waveform during volume control mode (a) to an airway pressure waveform with reverse-triggering effect (b) from the same patient at equal ventilator settings within three breaths. The reduced airway pressure is evident.

This study presents a simple model-based method capable of improving the consistency of identified respiratory mechanics in real-time. A pressure waveform reconstruction method was used to generate surrogates of SB 'unaffected' breathing cycles, to identify the 'true' underlying respiratory mechanics. In essence, this method seeks to recreate the pressure waveform obscured by SB. These 'unaffected' pressure waveforms can be used to estimate the patient-specific underlying respiratory mechanics in real-time, which can be used to guide MV therapy [10, 13, 14]. 


\subsection{Method}

\subsection{Respiratory System Mechanics Model}

Respiratory mechanics can be used to characterise patient-specific condition and response to treatment, and are conventionally estimated using a single-compartment linear lung model $[9,15]$.

$$
P_{a w}=E_{r s} \times V+R_{r s} \times Q+P 0
$$

$P_{a w}$ is the airway pressure, $E_{r s}$ is the respiratory system elastance, $V$ is the lung volume, $R_{r s}$ is the respiratory system resistance, $Q$ is the airway flow, and $P O$ is the offset pressure or positive end expiratory pressure (PEEP) if there is little or no intrinsic PEEP. Using easily measured inspiratory airway pressure and flow data, $E_{r s}$ and $R_{r s}$ can be estimated using linear regression [2, 4, 9].

\subsection{Pressure Reconstruction Method}

This method utilises a simple algorithm that superimposes consecutive breath waveforms, pooling breath data together. Despite the effect of SB efforts or reverse-triggering, each breathing cycle contains variable regions unaffected by $\mathrm{SB}$, as illustrated in Figure 2. Pooling by superimposing multiple breathing cycles can thus extend the region of 'unaffected' data, reduce noise effects and improve confidence in the unaffected data. A measurable portion of a seemingly unaffected breathing cycle can be reconstructed by overlaying affected pressure curves and taking the maximum or peak values. 

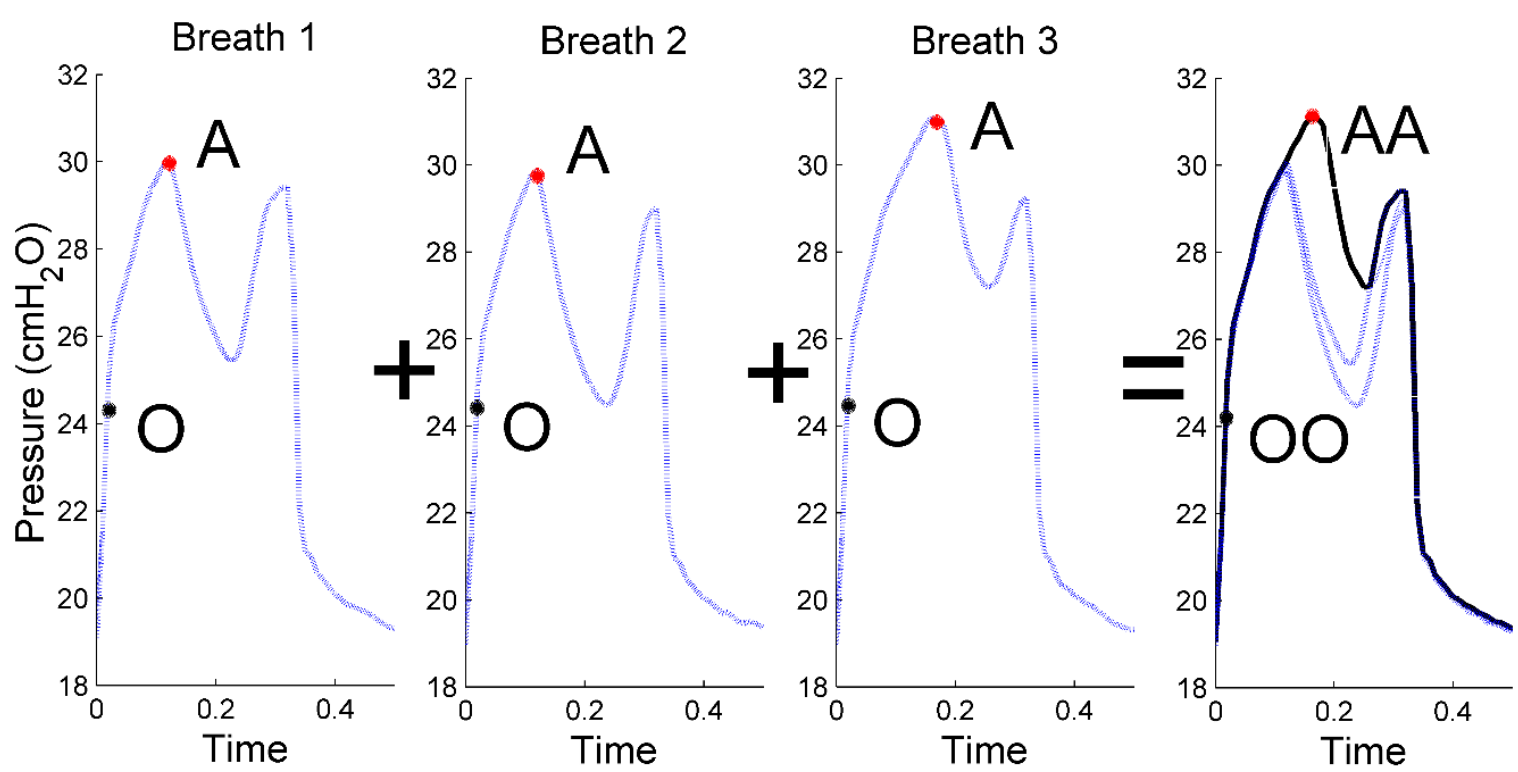

Figure 2. How pressure waveform reconstruction is used to reconstruct a breathing cycle with more 'correct' data from three clinical breathing cycles affected by reverse-triggering.

It is important to note that it may not be possible to reconstruct a full, unaffected waveform as with the example shown in Figure 2. Incomplete pressure reconstruction is due to the variability of SB magnitude and timing and the number of breaths used. Previous work has reported five breaths to be an optimal balance between efficacy and effort [16]. It is thus important to correctly identify the unaffected pressure waveform region. A Hamming windowed low-pass filter enables the identification of the point where the pressure gradient first becomes negative (Points A and AA of Figure 2). After Point A, the pressure waveform data is assumed to be compromised by patient induced SB unless reconstruction can prove otherwise. Figure 2 illustrates how pooling three breaths can build confidence in the initial region of inspiration and can lengthen O-A thus qualifying more data points to be considered during regression.

The reconstructed pressure waveform is also inspected for gradient sign changes to determine the unaffected region. A typical non-SB pressure waveform (Figure 1(a)) is expected to have one change in gradient sign (positive - negative) during inspiration. If the reconstructed pressure waveform has more than one change in slope (positive - negative - positive [- negative]) as in Figure 1(b), the reconstruction is classified as incomplete, as it still resembles a SB affected breath, and the point at the first sign change (Figure 2, point AA), is the last point unaffected by SB. Data from 0.1 seconds past 
the beginning of inspiration (Figure 2, point OO) to this point can then be used to identify respiratory mechanics.

\subsection{Patients and Analysis}

In this study, respiratory failure data containing SB or reverse-triggering from Christchurch Hospital ICU were used to test the performance of the proposed pressure reconstruction algorithm. These patients were ventilated using a Puritan Bennett 840 ventilator (Covidien, Boulder CO, USA), using Synchronous Intermittent Mandatory Ventilation (SIMV) mode delivering fixed tidal volume (6-8 $\mathrm{mL} / \mathrm{kg}$ ) in ramp flow. The airway pressure and flow data were collected using a bedside airway pressure, flow and respiratory mechanics monitoring tool connected to the ventilator [14]. The primary cause of respiratory failure of the patients are shown in Table 1. The collection and use of this data is approved by the New Zealand Southern Region Ethics Committee. Written informed consent was obtained from each patient.

Table 1. Primary cause of respiratory failure of each patient considered.

\begin{tabular}{|l|l|l|}
\hline RM No. & Cause of Respiratory Failure & Age and Sex \\
\hline $\mathbf{1}$ & Faecal peritonitis & $53 \mathrm{~F}$ \\
\hline $\mathbf{2}$ & Cardiac surgery and contracted hospital acquired pneumonia & $71 \mathrm{M}$ \\
\hline $\mathbf{3}$ & Pneumonia & $60 \mathrm{M}$ \\
\hline
\end{tabular}

In the data collection trial, respiratory failure patients in the ICU are subjected to stepwise recruitment manoeuvres (RMs) early in their ventilation to recruit collapsed lung volume. A key symptom of respiratory failure is non-aerated parenchyma, any improvement can facilitate oxygen perfusion to the blood, promoting oxygenation and outcomes [17]. Prior to a RM, patients are given muscle relaxants to suppress any SB effort and allow the patient's lung to adapt to the changes in MV PEEP settings, per clinical standard [17-19]. Airway pressure and flow data was collected, starting before muscle paralysis and continuing for 3 hours after the RM, as illustrated in Figure 3. 
To test the performance of the proposed pressure reconstruction algorithm, two regions (A and B in Figure 3) of pressure and flow data were considered for each RM. Specifically, Region A was before the RM where patient-specific SB effort or reverse-triggering was initially prevalent but subsequently diminished with the administration of muscle relaxants per local clinical protocol. Region B included 3 hours after the RM, during which time paralysing agents were metabolised, their effect weakened, and patient-specific SB efforts may reappear.

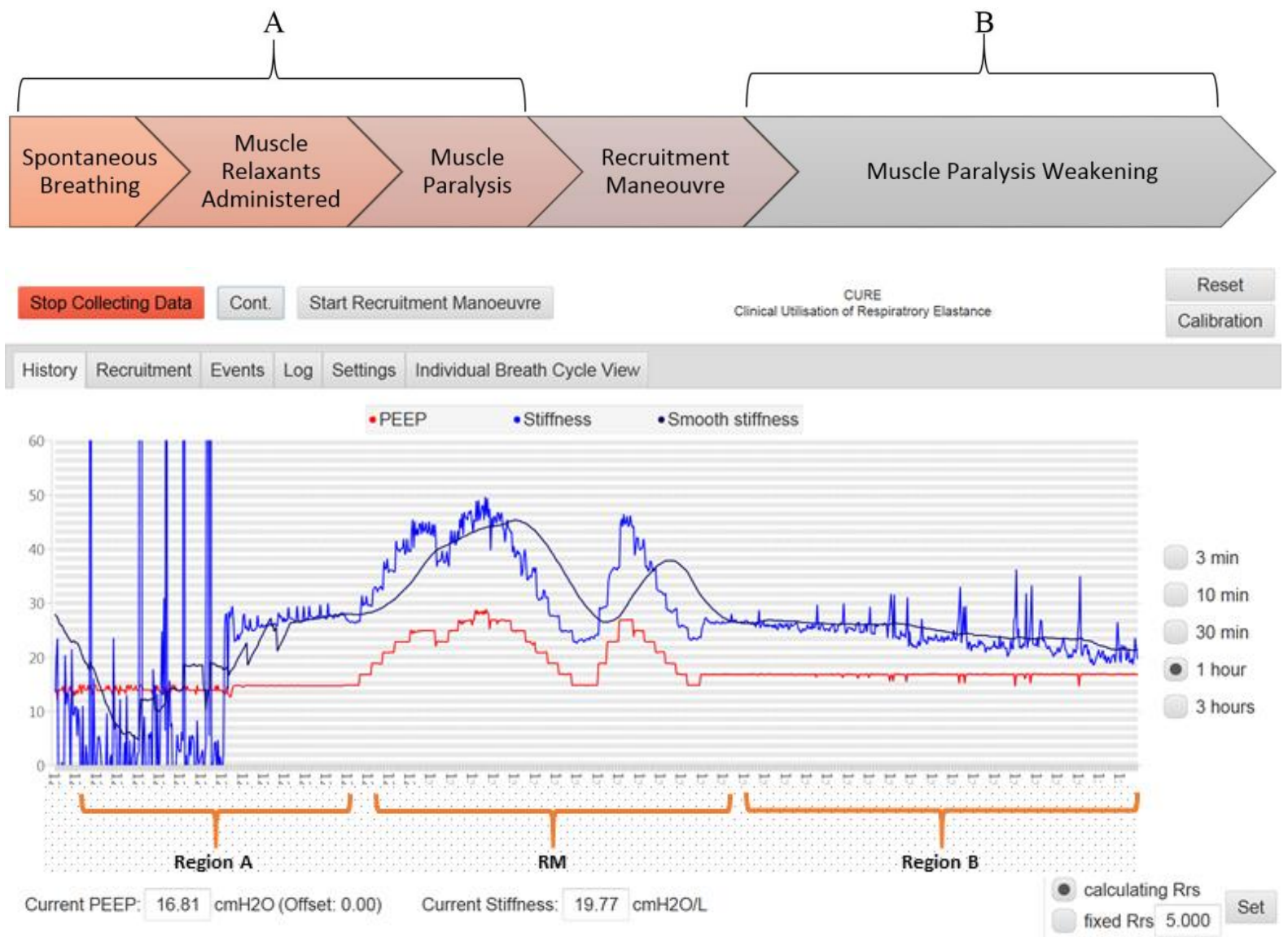

Figure 3. (a): timeline illustrating the progression of the study and the effect of muscle paralysis. (b): sample of the progression of the study [14].

Varying SB efforts causes an increase in breath-to-breath variability of identified respiratory elastance.

Conventional identification, by applying the single-compartment model over the entire inspiratory waveforms, regardless of the magnitude of SB, is thus expected to perform poorly during SB, but perform well during muscle paralysis. The pressure reconstruction method should match the conventional results during muscle paralysis while improving consistency during SB. Additionally, the 
pressure reconstruction method should perform consistently regardless of SB severity. Thus, Regions A and B will provide a platform to compare the performance of pressure reconstruction by comparing with the patient's 'true' respiratory mechanics estimated conventionally during muscle paralysis unaffected by SB - the patient acts as their own control over one RM.

\subsection{Analysis}

The performance of the pressure reconstruction algorithm is compared with conventional identification without reconstruction. Performance of the pressure reconstruction method is assessed by comparing the variability of identified $E_{r s}$ with and without pressure reconstruction, using a non-parametric coefficient of variation $(\mathrm{CV})$, which is defined as the ratio of the interquartile range [IQR] and the median [12].

Comparisons within Region A between the two methods pre- and post-paralysis were made using the Wilcoxon rank-sum (RS) test and the two-sample Kolmogorov-Smirnov (KS) test, and p $<0.05$ is considered significant. The four cases are illustrated by Figure 4 with the expected result for each case.

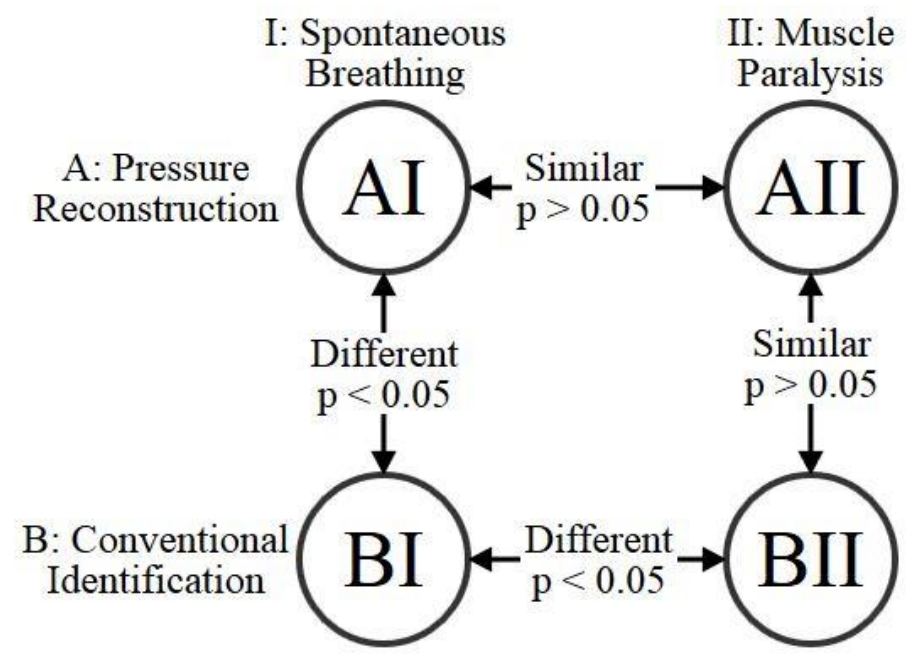

Figure 4. Schematic of the four cases of Region A and the hypothesised result of each comparison.

Ideally, pressure reconstruction should perform consistently during $\mathrm{SB}$ and muscle paralysis (AI is 
similar to AII, $\mathrm{p}>0.05$ ) and during muscle paralysis pressure reconstruction should be comparable to the conventional method (AII similar to BII, $\mathrm{p}>0.05$ ). However, since the conventional method is expected to perform poorly during SB we expect it to differ compared to pressure reconstruction (BI is different from AI, $\mathrm{p}<0.05$ ) and differ from itself during muscle paralysis (BI is different from BII, $\mathrm{p}<$ $0.05)$. 


\subsection{Results}

\subsection{Region A - before RM}

Figures 5-7 compare $E_{r s}$ estimation using conventional identification and pressure reconstruction in Region A for several cases, RM 1 - RM 3. Examples of airway pressure waveforms before and after muscle relaxants administration are also shown in these Figures. The median and IQR of the $E_{r s}$ and the corresponding CV before and after administration of muscle relaxants are shown in Tables 2 and 3.

(a)
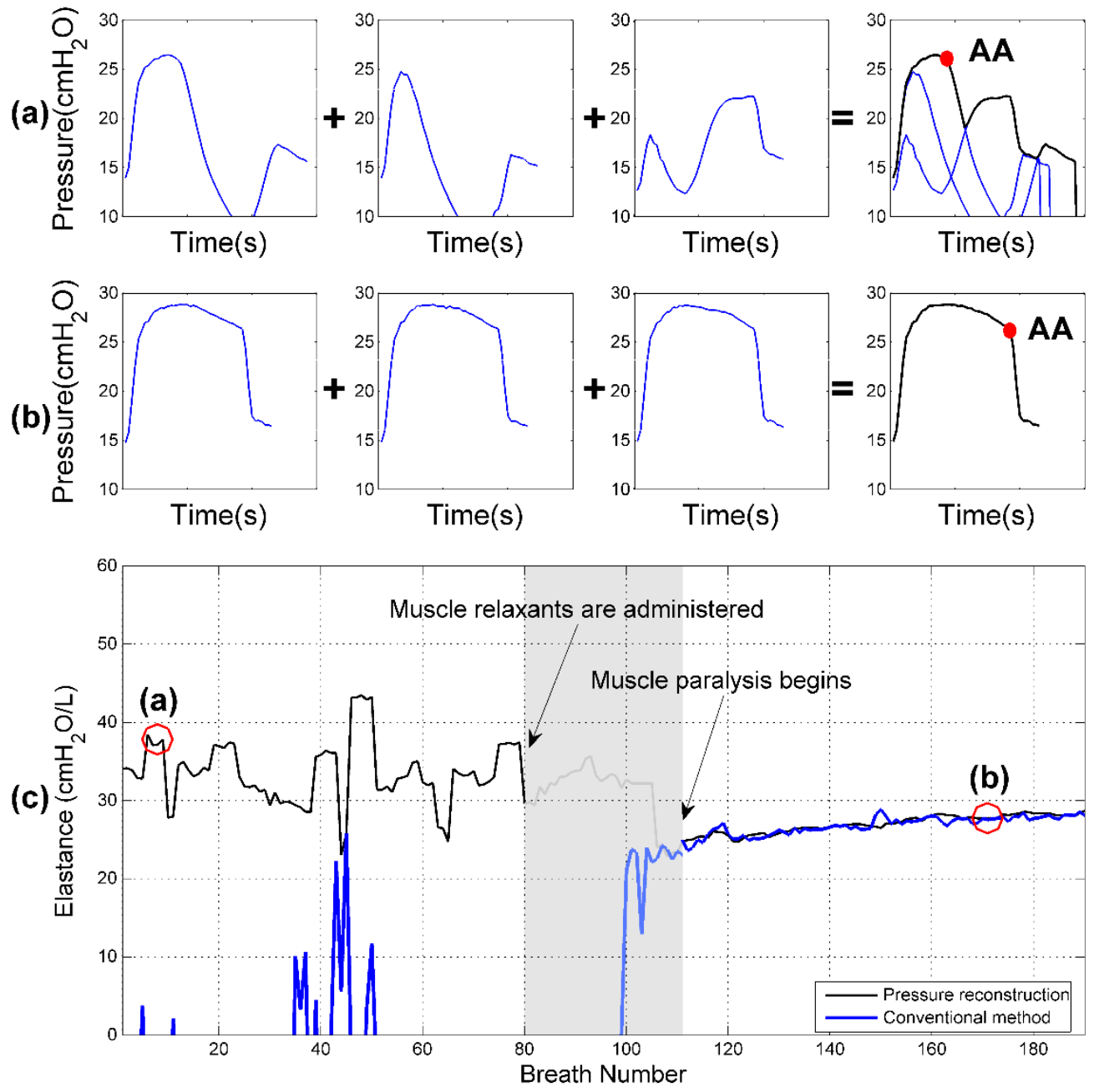

Figure 5. Pressure reconstruction and $E_{r s}$ for RM 1. (a): 3 consecutive airway pressure waveforms exhibiting spontaneous breathing efforts before paralysis are used for pressure reconstruction at time (a). (b): Muscle paralysis yielding typical airway pressure waveforms at time (b). (c): $E_{r s}$ before and after muscle paralysis for conventional identification and pressure reconstruction 

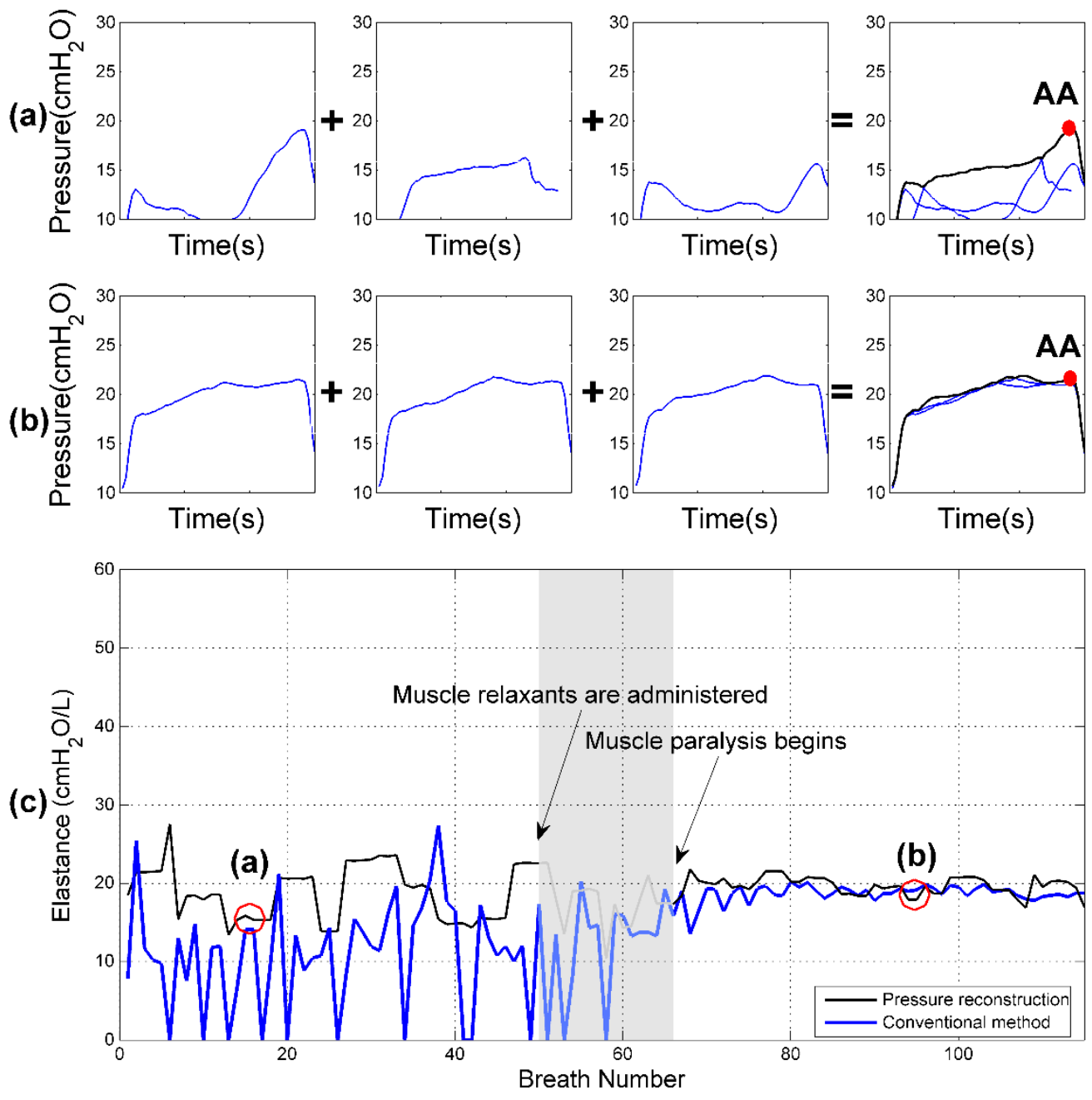

Figure 6. Pressure reconstruction and $E_{r s}$ for RM 2. (a): 3 consecutive airway pressure waveforms exhibiting spontaneous breathing efforts before paralysis are used for pressure reconstruction at time (a). (b): Muscle paralysis yielding typical airway pressure waveforms at time (b). (c): $E_{r s}$ before and after muscle paralysis for conventional identification and pressure reconstruction 
(a)
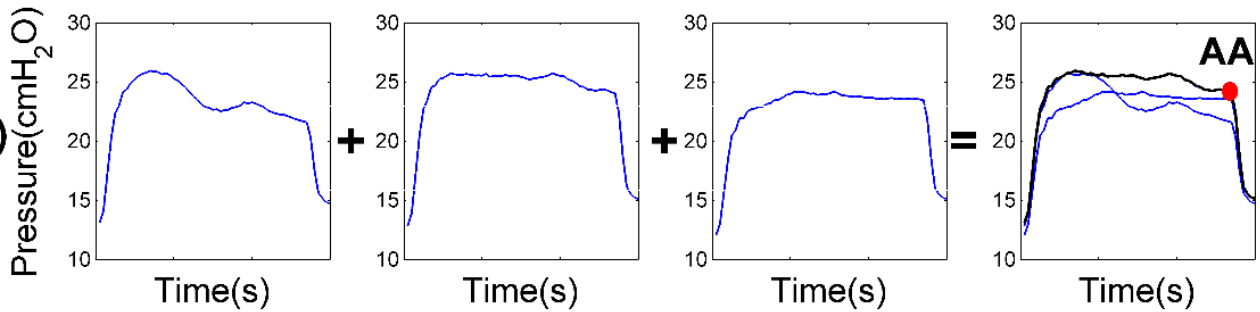

(b)
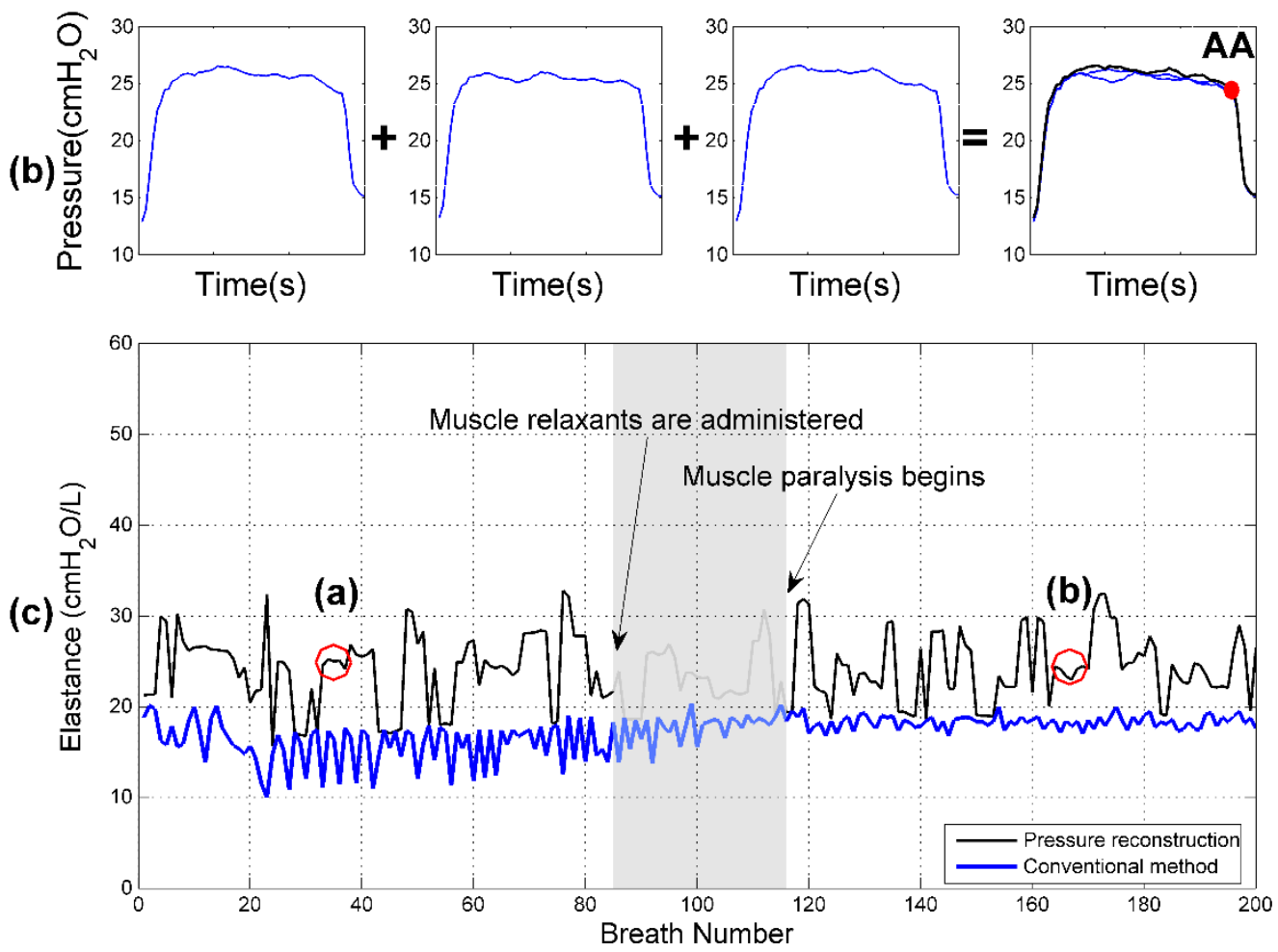

Figure 7. Pressure reconstruction and $E_{r s}$ for RM 3. (a): 3 consecutive airway pressure waveforms exhibiting spontaneous breathing efforts before paralysis are used for pressure reconstruction at time (a). (b): Muscle paralysis yielding typical airway pressure waveforms at time (b). (c): $E_{r s}$ before and after muscle paralysis for conventional identification and pressure reconstruction 
Table 2. Comparison of respiratory system elastance $\left(E_{r s}\right)$, in Median [IQR] $\mathrm{cmH}_{2} \mathrm{O} / \mathrm{L}$, during $\mathrm{SB}$ and paralysis portions of Region A with the corresponding RS and KS test results.

\begin{tabular}{|c|c|c|c|c|c|c|c|c|}
\hline & \multicolumn{2}{|c|}{ Before Paralysis } & & \multicolumn{2}{|c|}{ During Paralysis } & \multirow[b]{2}{*}{$\begin{array}{r}\text { AII/ } \\
\text { BII }\end{array}$} & \multicolumn{2}{|c|}{ Comparing Before to After } \\
\hline & $\begin{array}{c}\text { Conv. } \\
\text { BI }\end{array}$ & $\begin{array}{c}\text { Pres Rec. } \\
\text { AI }\end{array}$ & $\begin{array}{c}\mathrm{AI} / \mathrm{B} \\
\mathrm{I}^{\dagger}\end{array}$ & $\begin{array}{c}\text { Conv. } \\
\text { BII }\end{array}$ & $\begin{array}{c}\text { Pres Rec. } \\
\text { AII }\end{array}$ & & $\begin{array}{c}\text { Conv. } \\
\text { BI/BII*广 }\end{array}$ & $\begin{array}{l}\text { Pres Rec. } \\
\text { AI/AII }\end{array}$ \\
\hline RM 1 & $\begin{array}{c}-19.0 \\
{[-23.6--11.3]}\end{array}$ & $\begin{array}{c}33.3 \\
{[31.6-36.5]}\end{array}$ & $\begin{array}{l}* \\
\dagger\end{array}$ & $\begin{array}{c}27.0 \\
{[26.2-27.2]}\end{array}$ & $\begin{array}{c}27.0 \\
{[26.0-28.1]}\end{array}$ & & $\begin{array}{l}* \\
\dagger\end{array}$ & $\begin{array}{l}* \\
\dagger\end{array}$ \\
\hline RM 2 & $\begin{array}{c}11.8 \\
{[7.77-14.7]}\end{array}$ & $\begin{array}{c}19.4 \\
{[15.4-21.5]}\end{array}$ & $*$ & $\begin{array}{c}18.9 \\
{[18.4-19.3]}\end{array}$ & $\begin{array}{c}19.0 \\
{[15.3-21.6]}\end{array}$ & $*$ & $*$ & $\dagger$ \\
\hline RM 3 & $\begin{array}{c}16.4 \\
{[14.4-17.5]}\end{array}$ & $\begin{array}{c}24.9 \\
{[21.2-26.7]}\end{array}$ & $*$ & $\begin{array}{c}18.4 \\
{[17.8-18.9]}\end{array}$ & $\begin{array}{c}24.9 \\
{[21.2-26.7]}\end{array}$ & $*$ & $*$ & \\
\hline
\end{tabular}

Note: $*$ and ${ }^{\dagger}$ refer to cases where $\mathrm{p}<0.05$ for the RS and KS tests respectively; Conv. - Conventional identification; Pres Rec. - Pressure reconstruction.

The RM 3 patient before paralysis exhibits relatively little SB effort. It was observed that the airway pressure waveforms were similar before and during muscle paralysis, as illustrated in Figure 7. This patient was likely to have been heavily sedated and paralysed prior to the administration of additional muscle relaxant pre-RM.

Table 3. Comparison of respiratory system elastance $\left(E_{r s}\right)$ CV during SB and paralysis regions.

\begin{tabular}{|l|c|c|c|c|c|c|}
\hline & \multicolumn{3}{|c|}{ Before Paralysis with SB } & \multicolumn{3}{c|}{ During Paralysis } \\
\hline & $\begin{array}{c}\text { Conventional } \\
\text { Method }\end{array}$ & $\begin{array}{c}\text { Pressure } \\
\text { reconstruction }\end{array}$ & Difference & $\begin{array}{c}\text { Conventional } \\
\text { Method }\end{array}$ & $\begin{array}{c}\text { Pressure } \\
\text { reconstruction }\end{array}$ & Difference \\
\hline RM 1 & 0.6483 & 0.1451 & -0.5032 & 0.0582 & 0.0768 & +0.0186 \\
\hline RM 2 & 0.5839 & 0.3158 & -0.2681 & 0.0499 & 0.0975 & +0.0476 \\
\hline RM 3 & 0.1893 & 0.2239 & +0.0346 & 0.0585 & 0.2335 & +0.1750 \\
\hline
\end{tabular}




\subsection{Region B - after RM}

Figures 8-10 illustrate the box plots for the $E_{r s}$ estimated for 3000 breaths (2 3 hours) directly after the RM for the 3 RMs of Region A. Each box plot represents groups of 500 consecutive breaths during this time. Region B describes the trend of $E_{r s}$ as the patient metabolises the muscle relaxants and slowly recovers more severe SB efforts.

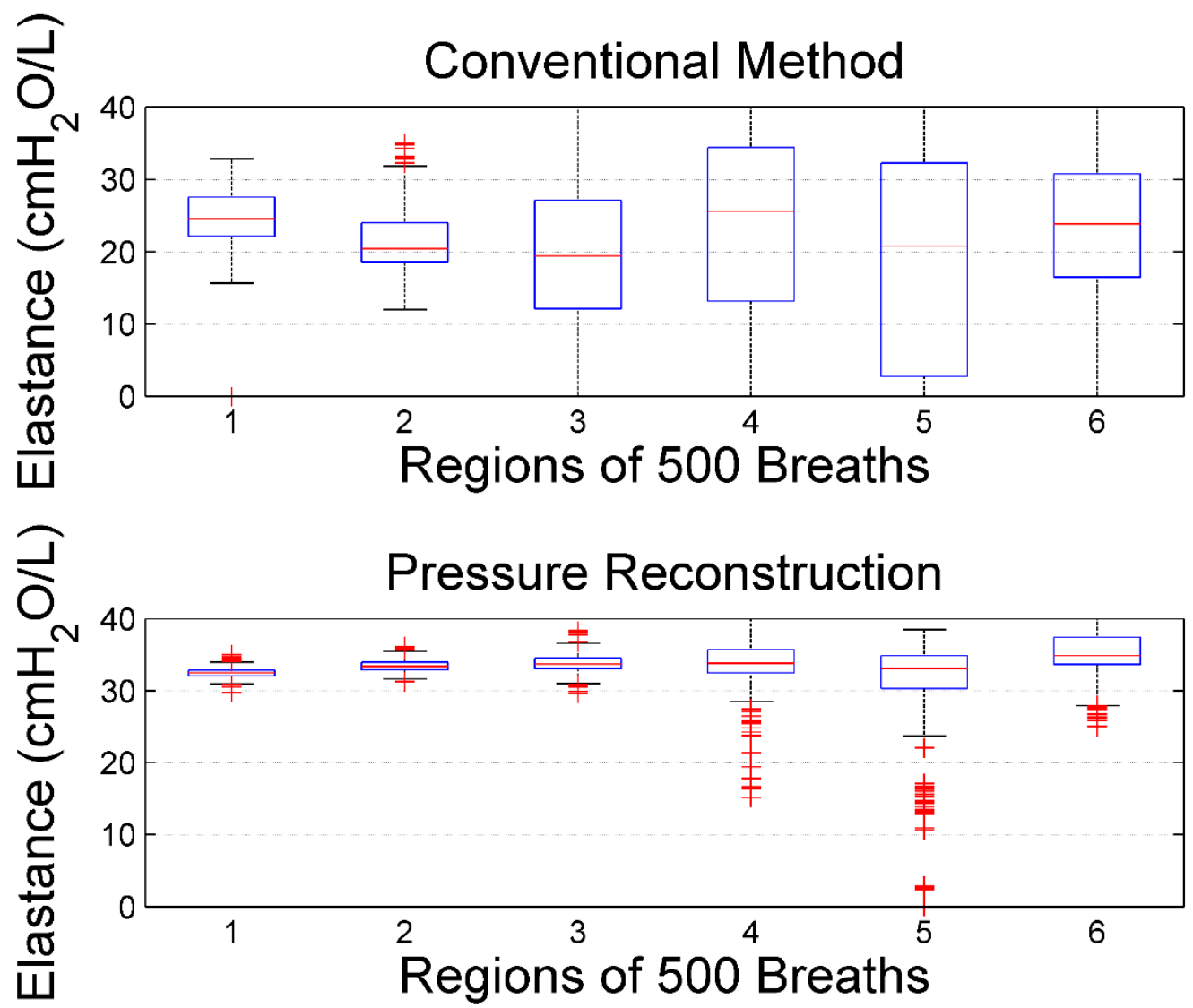

Figure 8. Respiratory system elastance $\left(E_{r s}\right)$ for RM 1. (a): Conventional method estimated $E_{r s}$ distribution. (b): Pressure reconstruction estimated $E_{r s}$ distribution. 

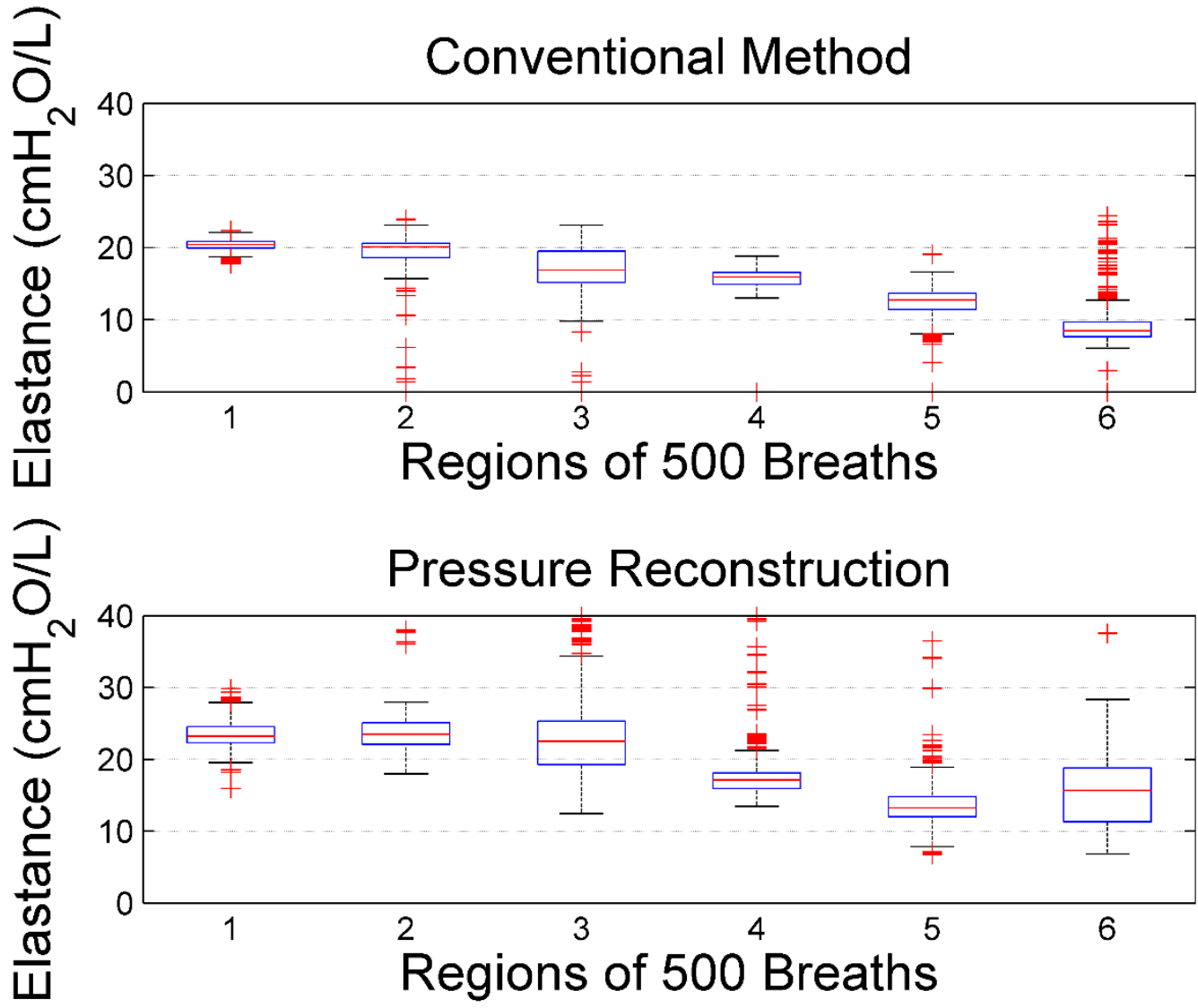

Figure 9. Respiratory system elastance $\left(E_{r s}\right)$ for RM 2. (a): Conventional method estimated $E_{r s}$ distribution. (b): Pressure reconstruction estimated $E_{r s}$ distribution. 


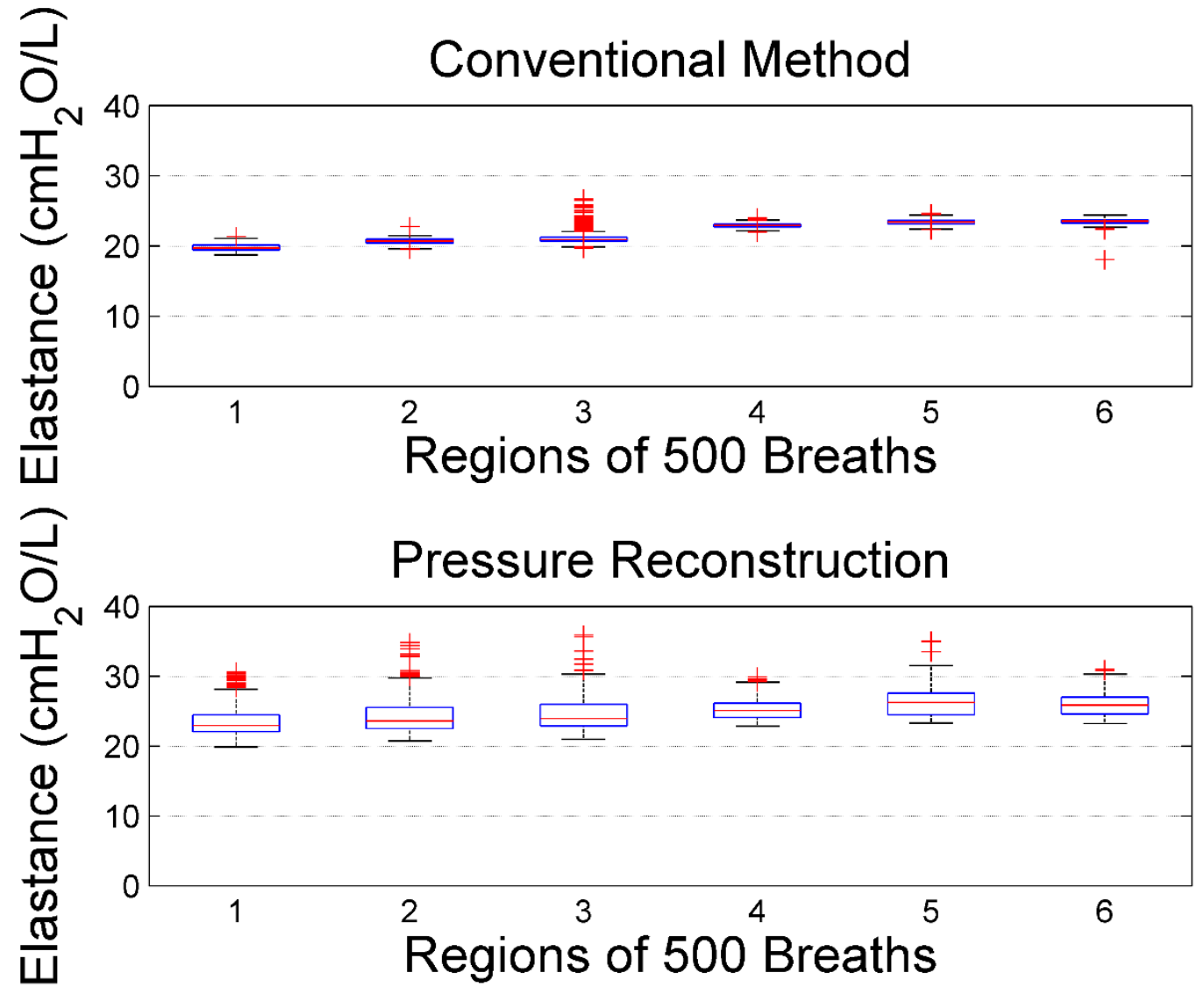

Figure 10. Respiratory system elastance $\left(E_{r s}\right)$ for RM 3. (a): Conventional method estimated $E_{r s}$ distribution. (b): Pressure reconstruction estimated $E_{r s}$ distribution.

KS tests applied to the results of conventional identification and pressure reconstruction in each of the six 500 breath regions as well as comparing each subsequent region described that for all three cases, each distribution is different $(\mathrm{p}<0.05)$. 


\subsection{Discussion}

\subsection{Region A: The effect of muscle relaxants}

Prior to muscle relaxant administration, for RMs 1 and 2, the $E_{r s}$ estimated from conventional identification yields non-physiological negative values with large fluctuations (Figures 5(c) and 6(c)). These fluctuations are caused by the highly atypical pressure waveforms of Figures 5 and 6(a). These waveforms do not emulate the typical airway pressure profile described in Figure 1(a) and thus, the single-compartment model cannot estimate $E_{r s}$ correctly.

When muscle relaxants are administered, the effect on patient SB is almost immediate. The pressure waveforms cease to show any SB effect, illustrated in Figure 5(b). The unaltered pressure waveform will allow a good estimation of respiratory mechanics using linear regression $[2,4,9]$. The patient's condition should not otherwise change during transition. Thus, the $E_{r s}$ estimated prior to paralysis should be similar, if not equal, to that estimated post-administration of muscle paralysis. The period from pre- to post-muscle paralysis provides the means of validating the $E_{r s}$ estimated using pressure reconstruction method with the true $E_{r s}$ during paralysis.

In this study, it was found that pressure reconstruction was able to estimate physiological $E_{r s}$ values during the period pre-paralysis similar to $E_{r s}$ post-paralysis, for example, Figure 5(c) near time (b) illustrates where $E_{r s}$ values from both methods are nearly identical. Table 3 indicates that reductions in $E_{r s}$ variability, as captured by $\mathrm{CV}$, are specific to the patient and the patient's condition. Reductions in CV range from 0.5032 (78\%) for RM 1 to 0.2681 (46\%), for RM 2 while for RM 3, the CV increased by $0.0346(18 \%)$. Table 2 shows that pressure reconstruction results are much more consistent (smaller IQR and $\mathrm{CV}$ ) and accurate (matching post-paralysis) estimates of $E_{r s}$ than conventional identification during severe SB.

Some results displayed in Table 2 do not agree with the expected results. Pressure reconstruction applied in RMs 2 and 3 during muscle paralysis causes a small increase in $E_{r s}$ using pressure reconstruction, as shown in Figures 6(c) and 7(c). This difference in median $E_{r s}$ yields $\mathrm{p}<0.05$ for the RS test comparing 
AII and BII without affecting the shape of the distributions $(\mathrm{p}>0.05)$. This disparity could possibly be due to effects of sedation or muscle paralysis on the chest-wall compliance by affecting the intercostal muscles. Alternatively, the susceptibility of pressure reconstruction to noise effects by extracting the maximum values of the pooled waveforms, could marginally increase the median estimated $E_{r s}$.

Compared to RMs 1 and 2, RM 3 (Figure 7) described a different response. In this case, conventional identification performed well prior to muscle relaxant administration due to fewer and less severe SB induced reverse triggering. Pressure reconstruction can do little to create a more consistent $E_{r s}$ estimation in this case, since the breaths were not significantly affected. Thus, the quantified effect of pressure reconstruction is poorer, with an increase in $\mathrm{CV}$ of $0.0346(18 \%)$. Hence, the pressure reconstruction method fails to yield a more consistent estimation of $E_{r s}$, and this issue remains a potential limitation of the approach.

When a patient is exhibiting severe $\mathrm{SB}$, such as RMs 1 and 2, the identified $E_{r s}$ by pressure reconstruction is greatly less variable than conventional identification. After paralysis, the variation is small for both methods and pressure reconstruction successfully recreates the median value of $E_{r s}$ for the patient at that time. 


\subsection{Region B - Metabolism of muscle relaxants}

In contrast to Region A where muscle paralysis takes effect very quickly, assessment of Region B requires a longer time period as the muscle relaxants are metabolised and cleared from the body over hours at a patient-specific rate dependent on the quantity administered and the patient's metabolic rate. As muscle paralysis weakens, if the patient respiratory rate is not synchronised with the ventilator, the effect of the patient's SB efforts increase and eventually rival the initial, before paralysis state.

Figures 8-10 illustrate the time-varying progression of SB returning, indicated by the decreasing median and increasing IQR of the $E_{r s}$ box plot. The time-varying evolution of $E_{r s}$ is non-linear but the general trend for both methods during RM 1 is a reasonably consistent median as the IQR generally increases. RM 2 differs as the median of $E_{r s}$ decreases while the IQR fluctuates but remains relatively low. RM 3 describes a small increase in IQR likely caused by the lack of severe SB. Visually, pressure reconstruction performs better than, or at minimum similar, to conventional identification as paralysis weakens.

During Region B for RM 2, the patient is breathing erratically and for clinical reasons the ventilator mode is changed several times. The breath duration fluctuates significantly, implying the switch from a full volume-control to an assisted volume-support mode. The sixth region of Figure 9 illustrates a large, sudden increase in the pressure reconstruction identified $E_{r s}$ caused by the change in ventilation mode and very rapid breathing. Since the mode changed to a support mode in region 6 , this portion of data is no longer valid for pressure reconstruction.

Each distinct 500 breath section of Region B using pressure reconstruction is compared to conventional identification of the same section and also compared to its preceding section. In all cases the KS test describes the distribution of each region to be different. Inspection of the time-varying data illustrates both high frequency fluctuations and varying trends. Each region captures estimations of $E_{r s}$ from a distinct portion of time thus yielding $\mathrm{p}<0.05$. Many complex factors are not included in the above analysis that can contribute to the patient's changes in Ers, far more than simply the metabolism of the 
muscle relaxants. Some examples of these complex factors include: 1) turning of the patient, which can alter breathing dynamics as well as the measured respiratory mechanics; 2) fluid build-up in the lungs or ventilator endotracheal tubes, which can distort the ventilator recorded pressure and flow data; 3) endotracheal suctioning; and 4) a sudden change of respiratory mechanics due to derecruitment of the lung following the RM which will stiffen the lung and report a higher $E_{r s}$ value.

In cases of severe SB affected breath waveforms, pressure reconstruction can significantly reduce the variation of $E_{r s}$ over three hours post RM. Similar to Region A, pressure reconstruction improves, or at minimum recreates, the accuracy and consistency of $E_{r s}$ estimations while muscle paralysis is weakening. 


\subsection{Limitations}

Patient condition in Table 1 was primarily a function of pneumonia requiring MV. It should be noted that patient-specific disease state does not affect the pressure reconstruction algorithm. The algorithm is suitable for any patient who exhibits spontaneous breathing efforts, and thus is independent of patientspecific condition.

Figures 5-7 visually depict the improved performance of the reconstruction method, as well as some of its shortcomings. The proposed reconstruction method can overcome underestimations of pressure but is susceptible to unusually high pressure waveforms skewing the entire estimation higher. Pressure reconstruction applied to RM 3, described by Figure 7, illustrates many occasions where the identified $E_{r s}$ peaks and falls. These cases are caused by the building effect of pressure reconstruction where the maximum pressure leads to an overestimated $E_{r s}$. It is thus important to consider noise that may undermine the reconstruction method.

It is important to note that this reconstruction method is currently limited to volume-control modes. Pressure control ventilation has consistent pressure waveforms and thus pressure reconstruction will have no effect. Therefore, reconstruction of SB affected flow profiles will be needed for pressure control ventilation. Flow reconstruction to improve respiratory mechanics estimation warrants further investigation.

Some of the recorded reduction in variability could be attributed to the increased median $E_{r s}$ of pressure reconstruction. By definition, as the median increases the $\mathrm{CV}$ will decrease. Additionally the method is susceptible to disproportionate improvement by excluding breaths that contain insufficient data points to estimate $E_{r s}$. These excluded cases will be the 'worst' SB affected breaths and exclusion may result in improved quality of the data and thus an improvement in variability. Exclusion of the most severe breaths occurs 1,2 and 0 times from the patient data considered and does not contribute significantly to the improvement of reconstruction. Else, superimposing more pressure waveforms to more completely 
reconstruct the 'true pressure waveform' would exploit the best waveforms for a greater period but would not be ideal for cases of rapidly changing patient condition or ventilation settings such as the changing PEEP during a RM. 


\subsection{Conclusion}

Mechanically ventilated patients who exhibit spontaneous breathing efforts during full control ventilator support may alter the airway pressure waveform recorded. This alteration results in under or overestimation of the patient's true $E_{r s}$. Conventional identification, using a single-compartment model, does not account for these patient efforts and thus an unaffected pressure waveform must be reconstructed for respiratory mechanics estimation. The proposed pressure reconstruction algorithm performs very well compared to conventional identification in cases of severe SB. The algorithm compensates for the presence of SB and successfully reconstructs less-affected pressure waveforms and thus reduces the variability of identified $E_{r s}$, allowing more accurate $E_{r s}$ estimation that can be used for decision-making support to guide patient-specific MV care.

\subsection{Acknowledgements}

The authors wish to thank the Health Research Council of New Zealand and eTIME IRSES EU FP7 grant for supporting this research. The authors would also like to thank the University of Canterbury's Department of Mechanical Engineering for facilitating this research as undergraduate course credit.

\subsection{Conflict of Interest}

The authors declare that there are no conflicts of interest. 


\subsection{References}

[1] S. Rees, C. Allerød, D. Murley, Y. Zhao, B. Smith, S. Kjærgaard, P. Thorgaard, S. Andreassen, Using physiological models and decision theory for selecting appropriate ventilator settings, Journal of Clinical Monitoring and Computing, 20 (2006) 421-429.

[2] Y.S. Chiew, J.G. Chase, G. Shaw, A. Sundaresan, T. Desaive, Model-based PEEP Optimisation in Mechanical Ventilation, BioMedical Engineering OnLine, 10 (2011) 111.

[3] A. Sundaresan, J.G. Chase, Positive end expiratory pressure in patients with acute respiratory distress syndrome - The past, present and future, Biomedical Signal Processing and Control, 7 (2011) 93-103.

[4] E. van Drunen, Y.S. Chiew, C. Pretty, G. Shaw, B. Lambermont, N. Janssen, J. Chase, T. Desaive, Visualisation of time-varying respiratory system elastance in experimental ARDS animal models, BMC Pulmonary Medicine, 14 (2014) 33.

[5] D. Talmor, T. Sarge, A. Malhotra, C.R. O'Donnell, R. Ritz, A. Lisbon, V. Novack, S.H. Loring, Mechanical Ventilation Guided by Esophageal Pressure in Acute Lung Injury, New England Journal of Medicine, 359 (2008) 2095-2104.

[6] L. Brochard, G. Martin, L. Blanch, P. Pelosi, F.J. Belda, A. Jubran, L. Gattinoni, J. Mancebo, V.M. Ranieri, J.-C. Richard, D. Gommers, A. Vieillard-Baron, A. Pesenti, S. Jaber, O. Stenqvist, J.-L. Vincent, Clinical review: Respiratory monitoring in the ICU - a consensus of 16, Critical Care, 16 (2012) 219.

[7] E. Akoumianaki, S.M. Maggiore, F. Valenza, G. Bellani, A. Jubran, S.H. Loring, P. Pelosi, D. Talmor, S. Grasso, D. Chiumello, C. Guérin, N. Patroniti, V.M. Ranieri, L. Gattinoni, S. Nava, P.-P. Terragni, A. Pesenti, M. Tobin, J. Mancebo, L. Brochard, The Application of Esophageal Pressure Measurement in Patients with Respiratory Failure, American Journal of Respiratory and Critical Care Medicine, 189 (2014) 520-531.

[8] E. Akoumianaki, A. Lyazidi, N. Rey, D. Matamis, N. Perez-Martinez, R. Giraud, J. Mancebo, L. Brochard, J.-C.M. Richard, Mechanical ventilation-induced reverse-triggered breaths: A frequently unrecognized form of neuromechanical coupling, CHEST, 143 (2013) 927-938.

[9] U. Lucangelo, F. Bernabè, L. Blanch, Lung mechanics at the bedside: make it simple, Current Opinion in Critical Care, 13 (2007) 64-72

[10] Y.S. Chiew, C. Pretty, P.D. Docherty, B. Lambermont, G.M. Shaw, T. Desaive, J.G. Chase, TimeVarying Respiratory System Elastance: A Physiological Model for Patients Who Are Spontaneously Breathing, PLoS ONE, 10 (2015) e0114847.

[11] C. Sinderby, P. Navalesi, J. Beck, Y. Skrobik, N. Comtois, S. Friberg, S.B. Gottfried, L. Lindstrom, Neural control of mechanical ventilation in respiratory failure, Nat Med, 5 (1999) 1433-1436.

[12] K. Moorhead, L. Piquilloud, B. Lambermont, J. Roeseler, Y. Chiew, J.G. Chase, J.-P. Revelly, E. Bialais, D. Tassaux, P.-F. Laterre, P. Jolliet, T. Sottiaux, T. Desaive, NAVA enhances tidal volume and diaphragmatic electro-myographic activity matching: a Range90 analysis of supply and demand, Journal of Clinical Monitoring and Computing, 27 (2013) 61-70.

[13] M.-C. Pintado, R. de Pablo, M. Trascasa, J.-M. Milicua, S. Rogero, M. Daguerre, J.-A. Cambronero, I. Arribas, M. Sánchez-García, Individualized PEEP Setting in Subjects With ARDS: A Randomized Controlled Pilot Study, Respiratory Care, 58 (2013) 1416-1423.

[14] A. Szlavecz, Y. Chiew, D. Redmond, A. Beatson, D. Glassenbury, S. Corbett, V. Major, C. Pretty, G. Shaw, B. Benyo, T. Desaive, J. Chase, The Clinical Utilisation of Respiratory Elastance Software (CURE Soft): a bedside software for real-time respiratory mechanics monitoring and mechanical ventilation management, BioMedical Engineering OnLine, 13 (2014) 140.

[15] J.H.T. Bates, Lung Mechanics: An Inverse Modeling Approach, Cambridge University Press, United States of America, New York, 2009.

[16] D.P. Redmond, V. Major, S. Corbett, D. Glassenbury, A. Beatson, A. Szlavecz, Y.S. Chiew, G.M. Shaw, J.G. Chase, Pressure reconstruction by eliminating the demand effect of spontaneous respiration (PREDATOR) method for assessing respiratory mechanics of reverse-triggered breathing cycles, in: 2014 IEEE Conference on Biomedical Engineering and Sciences (IECBES) 2014, pp. 332-337.

[17] E. Fan, M.E. Wilcox, R.G. Brower, T.E. Stewart, S. Mehta, S.E. Lapinsky, M.O. Meade, N.D. Ferguson, Recruitment Maneuvers for Acute Lung Injury, American Journal of Respiratory and Critical Care Medicine, 178 (2008) 1156-1163. 
[18] M.O. Meade, D.J. Cook, L.E. Griffith, L.E. Hand, S.E. Lapinsky, T.E. Stewart, K.J. Killian, A.S. Slutsky, G.H. Guyatt, A Study of the Physiologic Responses to a Lung Recruitment Maneuver in Acute Lung Injury and Acute Respiratory Distress Syndrome, Respiratory Care, 53 (2008) 1441-1449.

[19] S. Bennett, W.E. Hurford, When Should Sedation or Neuromuscular Blockade Be Used During Mechanical Ventilation?, Respiratory Care, 56 (2011) 168-180. 